\title{
$108 \mathrm{~Tb} / \mathrm{s}$ Transmission over $120 \mathrm{~km}$ of 7-Core Multicore Fiber Link with Integrated Cladding Pumped Multicore Amplifiers
}

Talha Rahman(1)*, Bernhard Spinnler(1), Stefano Calabro(1), Erik De Man ${ }^{(1)}$, Klaus Pulverer(1), Bernd Sommerkorn-Krombholz(1), Carlos Castro(2), Takayuki Mizuno(3), Yutaka Miyamoto(3), Katsuhiro Takenaga $^{(4)}$, Saurabh Jain(5), Yongmin Jung( ${ }^{(5)}$, Shaif-ul Alam ${ }^{(5)}$ and Toshio Morioka(6)

(1) Coriant R\&D GmbH, St.-Martin-Str. 76, 81541 Munich, Germany, *talha.rahman@coriant.com

(2) Chair of Communications, Christian-Albrechts-Universität zu Kiel, Germany

(3) NTT Network Innovation Laboratories, NTT Corporation, 1-1 Hikari-no-oka, Yokosuka, Japan

(4) Advanced Technology Laboratory, Fujikura Ltd., 1440, Mutsuzaki, Sakura, Chiba, Japan

(5) Optoelectronics Research Centre, University of Southampton, Southampton, SO17 1BJ, UK

(6) Department of Photonics Engineering, Technical University of Denmark, Lyngby, Denmark

Abstract By employing time-domain hybrid modulations we maximize the throughput over a multicore fiber link. Modulation scheme ratio is adjusted according to the available OSNR at each spatial and wavelength channel achieving a net spectral efficiency of $39.27 \mathrm{bit} / \mathrm{s} / \mathrm{Hz}$ after $2 \times 60 \mathrm{~km}$ transmission.

\section{Introduction}

Multicore fiber transmission systems are very attractive for throughput-hungry applications like inter- and intra- data center connections. In these systems cost and power savings can be realized by using integrated multicore cladding pumped amplifiers ${ }^{1}$. Cladding pumped multicore amplifiers require only one multimode laserdiode for amplification of all cores, making them relatively smaller in size and attractive for commercial applications².

In order to minimize cost per transmitted bit, data-rate over a given link should be maximized. Employing coherent optical transmission technology, line rates in excess of $200 \mathrm{~Gb} / \mathrm{s}$ per wavelength can be achieved by classical modulation formats such as e.g. 16QAM, 32QAM, 64QAM, etc. ${ }^{2}$. To maximize the throughput over a given multicore transmission system, the order of the QAM formats can be adapted for each wavelength channel as well as for each core according to the available SNR at the receiver. However, classical modulation formats exhibit large differences in required signal-to-noise ratio (SNR) among each other. Hence, by using classical modulation formats, only a coarse optimization of the throughput over multicore transmission systems can be achieved. Advanced modulation techniques as e.g. time-domain hybrid QAM (TDHQ $)^{3-5}$, frequency-domain hybrid modulation $^{6}$, constellation shaping and multi-dimensional modulation formats provide much finer granularity in required SNR and achievable data-rates, keeping symbol rate and code rate fixed. By employing advanced modulation formats, throughput can be precisely maximized and cost per bit can be further reduced.

This paper demonstrates the use of TDHQ to maximize throughput over a $120 \mathrm{~km}$ long multicore transmission link with cladding pumped multicore fiber amplifiers.

\section{Experimental Setup}

Fig. 1 shows the experimental multi-core transmission setup. The multi-core link, embedded in a recirculating loop, consists of one $60-\mathrm{km}$ span of 7-core fiber and two cladding-pumped erbium-ytterbium-doped 7core fiber amplifiers (MC-EYDFA). Fiber fanin/fan-out couplers based on free-space optics are used to integrate the multi-core link in the

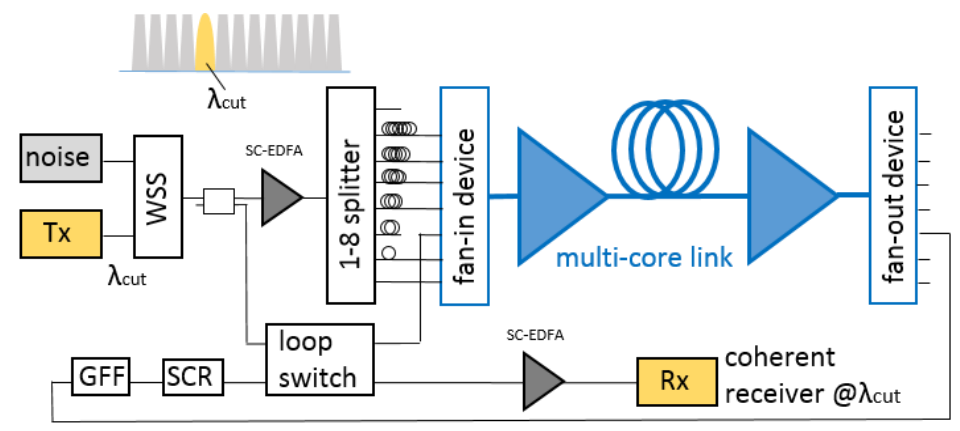

Fig. 1: Experimental setup. WSS: wavelength selective switch, GFF: gain flattening filter, SCR: pol. scrambler

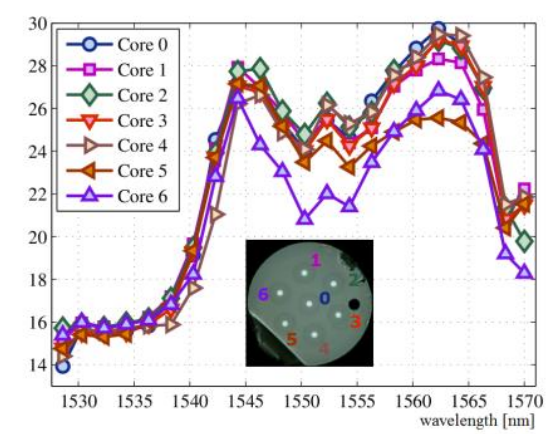

Fig. 2: Overall gain profile of the cascaded multi-core EYDFAs and multi-core fiber. Inset: A microscopic image of 7-core fiber. 
recirculating loop. The pump currents for both amplifiers are optimized by considering best performance for middle core as well as for two outer cores and taking the average value ${ }^{3}$.

The amplification band of the MCF amplifier covers the wavelengths $1566.723 \mathrm{~nm}$ to $1544.924 \mathrm{~nm}$ (or $191.35 \mathrm{THz}$ to $194.05 \mathrm{THz}$ ) making a $2.75 \mathrm{THz}$ frequency band available for transmission, corresponding to 55 channels at $50 \mathrm{GHz}$ channel spacing. The gain profile for these channels through the different cores over the complete link is shown in Fig. 2. Due to large difference in gain over wavelength and cores, the OSNR after transmission is also different for each wavelength and spatial channel. In such a case, using a single modulation format with fixed symbol-rate and code rate can only provide suboptimal throughput.

The input optical signal in each core consists of a channel under test (CUT) multiplexed with ASE noise to emulate wavelength division multiplexed (WDM) transmission ${ }^{7}$. CUT and ASE are multiplexed employing a wavelength selective switch (WSS) with $50 \mathrm{GHz}$ per channel bandwidth. Prior to input, WDM optical signal of each core is properly de-correlated by applying different delays in their optical path. In order to evaluate performance over different wavelength channels, Tx/Rx lasers as well as WSS are tuned at the desired wavelength.

The loop setup is so configured that the optical signal circulates twice through the core under test emulating $120 \mathrm{~km}$ transmission link. The optical loop consists of a gain flattening filter as well as a polarization scrambler to balance the optical spectrum and evenly distribute polarization dependent effects, respectively. Apart from core under test, the optical signals from all the neighboring cores are passively terminated after fan-out. After transmission over $120 \mathrm{~km}$ (two loops) of 7-core fiber, the BER was evaluated for all cores and wavelengths. After demodulation using an integrated coherent receiver, the signal was sampled with a $23-\mathrm{GHz}$ bandwidth real-time oscilloscope. The sampled signal was evaluated by offline DSP processing, which consists of $\mathrm{CD}$ equalization, carrier frequency and phase recovery, polarization demultiplexing, channel equalization, clock recovery and BER evaluation.

\section{Measurements, Results and Discussion}

The signal samples of the CUT were generated offline. The generated symbol stream was upsampled to 2 samples per symbol and spectrally shaped using a root-raised cosine filter with roll- off 0.2. On top of the payload data, the CUT includes $4 \%$ OTN overhead, 3\% overhead for training and pilot symbols, and $25 \%$ FEC overhead. The gross symbol rate was kept constant at $35 \mathrm{GBd}$ in order to exploit the full analog bandwidth of the components.

The received OSNR after $120 \mathrm{~km}$ transmission was in range of $21 \mathrm{~dB}$ to $26 \mathrm{~dB}$. Based on the available OSNR for each spatial and wavelength channel, TDHQ format was selected from a defined set. In this analysis we used 64QAM and 16QAM modulations in time domain hybrid frame. By controlling the ratio of 64QAM symbols to 16QAM symbols in time domain hybrid frame, the net data-rate for each channel could be configured quasi continuously between the two extremes $209 \mathrm{~Gb} / \mathrm{s}$ (16 QAM only) and $314 \mathrm{~Gb} / \mathrm{s}$ (64 QAM only). While in principle many more formats could be supported, in the experiment we used only the ratios of (64QAM, $16 \mathrm{QAM})=\{(90 \%, 10 \%)(80 \%, 20 \%)(67 \%, 33 \%)$ $(50 \%, 50 \%)(33 \%, 67 \%)$ (25\%, 75\%)\}. The back to back performance curves for the underlined cases are shown in Fig. 3. BER performance

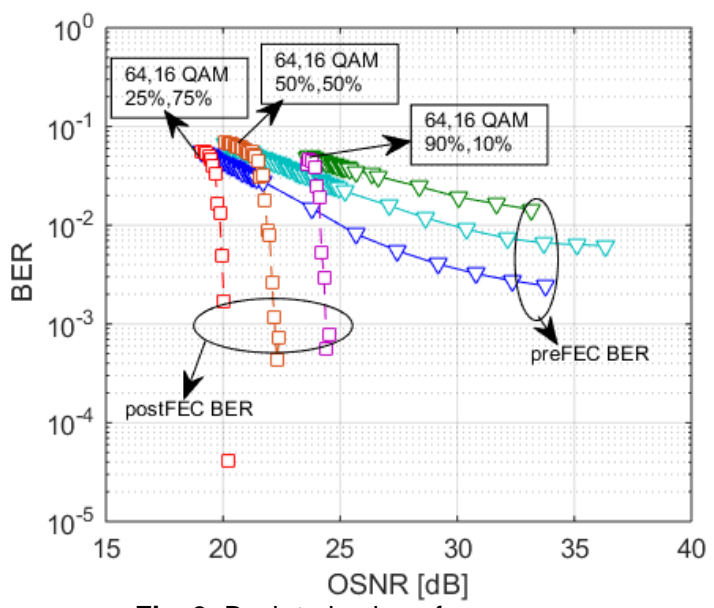

Fig. 3: Back-to-back performance

before FEC as well as after 20 FEC iterations are shown. It can be observed that required OSNR for error free performance of considered formats lies in range of $>20.5 \mathrm{~dB}$ to $>25.2 \mathrm{~dB}$ which coincides well with the available OSNR after $120 \mathrm{~km}$ transmission.

In order to select an appropriate scheme to be used at a given core and wavelength, first a monitoring signal is sent at the CUT wavelength to determine the received SNR. The modulation scheme ratio was then adjusted such that preFEC BER was expected slightly below the FEC threshold. Following this procedure for all wavelengths and cores, always maximum datarate for each channel was transmitted. Consequently, data throughput over the whole link was optimally maximized. 


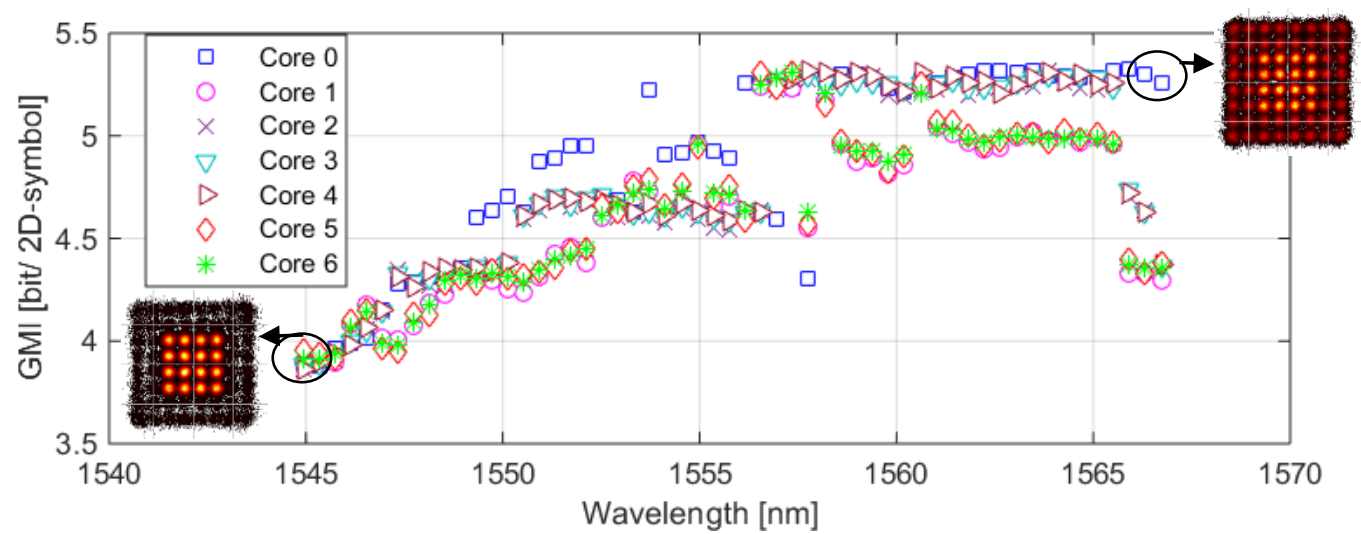

Fig. 4: Generalized mutual information for each wavelength and core after transmission over $120 \mathrm{~km}$. Inset: Constellation diagrams for 64QAM $/ 16 \mathrm{QAM}=(90 \%, 10 \%)$ and $(25 \%, 75 \%)$ cases.

After transmission through the link, all the wavelength and spatial channels were detected error-free employing 20 FEC iterations. The pre FEC BER for different channels lie in range of $3.1 \times 10^{-2}$ to $4.5 \times 10^{-2}$. The generalized mutual information $(\mathrm{GMI})^{8}$ for all the transmitted channels is evaluated after $120 \mathrm{~km} \mathrm{MCF}$ transmission and the corresponding results are shown in Fig. 4. It can be observed that larger throughput for all cores was achieved at longer wavelengths. Furthermore, cumulatively, the central core (core 0 ) could carry larger throughput than the rest because of larger gain for this core from the amplifiers. The net throughput for each core after $120 \mathrm{~km}$ transmission is summarized in table 1.

Tab. 1: Net data throughput for each core after

\begin{tabular}{|c|c|}
\hline Core & Net data throughput [Tb/s] \\
\hline 0 & 15.76 \\
\hline 1 & 15.13 \\
\hline 2 & 15.48 \\
\hline 3 & 15.48 \\
\hline 4 & 15.48 \\
\hline 5 & 15.13 \\
\hline 6 & 15.13 \\
\hline Total & 107.59 \\
\hline
\end{tabular}

\section{Conclusions}

We optimized data throughput over a multicore fiber link with integrated cladding pumped amplifiers employing suitable time domain hybrid modulation schemes after sensing received SNR for each channel. Consequently, a net data throughput of $107.59 \mathrm{~Tb} / \mathrm{s}$ was transmitted over a $2 \times 60 \mathrm{~km}$ link. In each core, 55 WDM channels with $50 \mathrm{GHz}$ spacing were transmitted which translates to a net spectral efficiency of $39.27 \mathrm{bit} / \mathrm{s} / \mathrm{Hz}$.

\section{Acknowledgements}

We would like to acknowledge support from Coriant. This work has been performed in the framework of the CELTIC EUREKA project SENDATE-FICUS (Project ID C2015/3-5), and it is partly funded by the German BMBF (Project ID 16KIS0487K). We would like to thank the project SAFARI for MC fiber and amplifiers.

\section{References}

[1] S. Jain et al., "32-core Inline Multicore Fiber Amplifier for Dense Space Division Multiplexed Transmission Systems," ECOC 2016 - PDP

[2] C. Castro et al., "100-Gb/s Transmission Over a 2520 km Integrated MCF System Using Cladding-Pumped Amplifiers," in IEEE PTL, vol. 29, no. 14, p. 1187 (2017)

[3] C. Castro, et al., "50 ch x 250 Gbit/s 32-QAM transmission over a fully integrated 7-core multicore link," ECOC 2017, M.1.E.5

[4] V. Curri et al., Time-division hybrid modulation formats: Tx operation strategies and countermeasures to nonlinear propagation, OFC 2014, no. Tu3A.2.

[5] Q. Zhuge, et al., Time Domain Hybrid QAM Based Rate-Adaptive Optical Transmissions Using High Speed DACs, OFC 2013, no. OTh4E.6.

[6] T. Rahman, et al., "Flexible Data-rate and Reach Transmission Employing Hybrid Modulation and Scrambled Coherent Superposition”, ECOC2017, Th.1.E.2

[7] D. J. Elson, et al., "Investigation of bandwidth loading in optical fibre transmission using amplified spontaneous emission noise," Opt. Express 25, 19529-19537 (2017)

[8] J. Cho et al., "Normalized Generalized Mutual Information as a Forward Error Correction Threshold for Probabilistically Shaped QAM", ECOC 2017, no. M.2.D.2. 